\title{
11 Mathematikdidaktisches Wissen angehender Lehrpersonen
}

Christian Brühwiler, Benita Affolter und Margit Kopp

\begin{abstract}
Dem fachdidaktischen Wissen kommt für die Gestaltung eines lernförderlichen Unterrichts eine hohe Bedeutung zu. Deshalb ist bei TEDS-M neben dem Fachwissen in Mathematik auch das mathematikdidaktische Wissen der angehenden Lehrpersonen gemessen worden. Die Ergebnisse zeigen für die Deutschschweiz ein insgesamt positives Bild über das mathematikdidaktische Wissen der angehenden Lehrpersonen. Sowohl die Leistungen der künftigen Lehrpersonen der Primarstufe als auch der Sekundarstufe I liegen signifikant über dem internationalen Mittelwert. Die Leistungsunterschiede zwischen den Lehrerbildungsinstitutionen der Deutschschweiz fallen in der Mathematikdidaktik zwar etwas geringer aus als beim mathematischen Wissen, sind aber zwischen den Primarstufenausbildungen teilweise beachtlich. Signifikante Geschlechterunterschiede lassen sich hingegen keine finden.
\end{abstract}

\subsection{Einleitung und Fragestellungen ${ }^{1}$}

Gegenwärtig wird in der Lehrerbildung die Diskussion um das richtige Verhältnis zwischen den Fachwissenschaften und den Fachdidaktiken engagiert geführt. ${ }^{2}$ Dabei haben die Fachdidaktiken in den vergangenen Jahren zunehmend an Autonomie gewonnen (Heitzmann, 2013) und sind im Begriff, sich gegenüber den Fachwissenschaften zu etablieren (Parchmann, 2013). Das neu gewonnene Selbstverständnis der Fachdidaktiken äussert sich beispielsweise an einer zunehmenden fachdidaktischen Forschungstätigkeit, aber auch darin,

1 Einzelne Teile dieses Kapitels sind bereits ähnlich in einem Online publizierten Bericht mit ersten Ergebnissen aus TEDS-M erschienen: Oser, F., Biedermann, H., Brühwiler, C., Kopp, M., Krattenmacher, S. \& Steinmann, S. (2010). Wie gut werden unsere angehenden Lehrpersonen ausgebildet? Ein internationaler Vergleich. Online unter: http://www.teds-m.ch/download/Erste_Ergebnisse_110222.pdf (17.10.2013).

2 Zum Beispiel das Themenheft „Fachdidaktik - Überlegungen und Standpunkte“ der Beiträge zur Lehrerbildung (Heitzmann et al., 2013). 
dass an verschiedenen Universitäten im deutschsprachigen Raum neue Fachdidaktik-Lehrstühle eingerichtet wurden. Auch wurde 2012 in der Schweiz ein eigener Dachverband „Konferenz Fachdidaktiken Schweiz“ (KOFADIS) gegründet, der sich u.a. für die Förderung der fachdidaktischen Aus- und Weiterbildung, die fachdidaktische Forschung und den wissenschaftlichen Nachwuchs in den Fachdidaktiken einsetzt.

Die wachsende Bedeutung der Fachdidaktiken für die Lehrerausbildung lässt sich auch empirisch begründen. So hat die COACTIV-Studie (Kunter et al., 2011) nachweisen können, dass das (schulnahe) Fachwissen von Mathematiklehrpersonen nicht direkt, sondern vermittelt über fachdidaktisches Wissen zu Leistungszuwächsen führen (Baumert et al., 2010; Baumert \& Kunter, 2011). Für die Gestaltung kognitiv aktivierender und motivierender Lerngelegenheiten bedürfe es insbesondere über ein hohes fachdidaktisches Wissen der Lehrperson. Baumert und Kunter (2011) kommen zum Schluss, dass fachdidaktisches Wissen die Unterrichtsqualität und den Lernzuwachs der Schülerinnen und Schüler stärker beeinflusst als das Fachwissen. Ein hohes methodischdidaktisches Wissen wird auch von Darling-Hammond und Youngs (2002) als wesentliches Merkmal erfolgreicher Lehrpersonen erachtet. Für einen gelingenden Unterricht ist nicht einfach der Umfang der verfügbaren Methoden massgebend, sondern die Lehrpersonen sollen vielmehr eine situationsgerechte Auswahl treffen und adaptiv einsetzen (Kunter \& Klusmann, 2010).

Grob gesagt geht es bei der Fachdidaktik um die Auswahl von Lerninhalten und das Wie des Unterrichtens, d.h. um fachspezifische Prinzipien und Regeln, die sowohl bei der Unterrichtsvorbereitung als auch bei der Durchführung von Unterricht wesentlich sind (Helmke, 2003). Lehrpersonen mit einem reichhaltigen Repertoire an didaktischen Gestaltungsformen haben bessere Möglichkeiten, ihren Unterricht adaptiv zu gestalten und flexibel auf Unvorhergesehenes zu reagieren (Brühwiler, 2014).

In Anlehnung an Shulman (1987) sowie Fan und Cheong (2002) kann mathematikdidaktisches Wissen in die beiden Teilbereiche curriculares und planungsbezogenes Wissen sowie interaktionsbezogenes Wissen aufgeteilt werden. Das curriculare und planungsbezogene Wissen beinhaltet die Kenntnis von Lehrplänen, darin enthaltene zentrale Themen zu identifizieren und $\mathrm{Zu}-$ sammenhänge $\mathrm{zu}$ erkennen, aber auch Lernziele $\mathrm{zu}$ formulieren und unterschiedliche Bewertungsformen zu kennen. Weiter gehören Kenntnisse und Fähigkeiten zur konkreten Planung des Fachunterrichts, in diesem Fall der Mathematik, dazu (Vollrath, 2001; Döhrmann, Kaiser \& Blömeke, 2010). Der Teilbereich des interaktionsbezogenen Wissens bezieht sich auf die Realisierung des Unterrichts, beispielsweise auf die Diagnosefähigkeit zur Interpretation und Bewertung von Schülerarbeiten, auf Kenntnisse und Fähigkeiten zur Führung von Unterrichtsgesprächen sowie auf die Erklärung mathematischer Sachverhalte.

Angesichts der hohen Bedeutung des fachdidaktischen Wissens von Lehrpersonen für gelingendes Unterrichten ist es naheliegend, dass deren Vermitt- 
lung zu den Kernaufgaben der Lehrerausbildung zählt. Allerdings gilt es auch festzuhalten, dass Lehramtsstudierende nicht nur fachliches, sondern zumindest implizit auch fachdidaktisches Wissen bereits vor der Lehrerausbildung erwerben und als Eingangsvoraussetzungen ins Studium mitbringen. In Kapitel 10.1 wurde argumentiert, dass sich mit Bezug auf die Fachwissenschaften zwei grundlegend verschiedene Modelle der Lehrerausbildung unterscheiden lassen, nämlich das Vorbildungs- und das Ausbildungsmodell. Dabei geht das Vorbildungsmodell davon aus, dass das fachwissenschaftliche Wissen weitgehend vor der Lehrerausbildung erworben wird, während im Ausbildungsmodell auch während des Studiums substanzielle Lerngelegenheiten angeboten werden, da das Vorwissen mit Blick auf die berufliche Praxis als nicht ausreichend beurteilt wird. Für die Fachdidaktik dürfte das Vorbildungsmodell eine untergeordnete Rolle spielen, weil in der Regel nicht davon ausgegangen werden kann, dass vor der Lehrerausbildung in ausreichendem Masse spezifische Kenntnisse im Bereich der Fachdidaktik erworben worden sind. Dementsprechend werden an allen Ausbildungstypen und -gängen in der Deutschschweiz mathematikdidaktische Lerngelegenheiten angeboten (vgl. Kapitel 6 und Kapitel 7 in diesem Band). Die vorhandenen zeitlichen Ressourcen werden also, besonders in der Primarlehrpersonenausbildung, die bezüglich Mathematik dem Vorbildungsmodell zugerechnet werden kann, stärker für die Auseinandersetzung mit Mathematikdidaktik als für eine fachwissenschaftliche Vertiefung eingesetzt.

Für den Erwerb mathematikdidaktischen Wissens sind neben den zur Verfügung stehenden Lerngelegenheiten auch individuelle Merkmale bedeutsam. Im vorliegenden Beitrag interessiert deshalb auch, ob mathematikdidaktisches Wissen mit soziodemografischen Merkmalen wie Geschlecht oder sozioökonomischer Hintergrund oder mit motivationalen Orientierungen zusammenhängt. Die internationalen Befunde aus TEDS-M verweisen darauf, dass sowohl fachbezogene wie altruistisch-pädagogisch Motive einen positiven Zusammenhang mit mathematikdidaktischen Leistungen aufweisen. Extrinsische Motive hängen, wie schon hinsichtlich der Mathematik (vgl. auch Kapitel 10 in diesem Band), auch mit dem mathematikdidaktischen Wissen negativ zusammen (Blömeke, Suhl, Kaiser \& Döhrmann, 2012). In diesem Kapitel werden Ergebnisse berichtet, die auf der Grundlage einer in der Deutschschweiz erweiterten Erfassung motivationaler Aspekte beruht (vgl. Kapitel 4 in diesem Band).

\section{Fragestellungen}

Eines der Kernanliegen von TEDS-M ist zu messen, über welches mathematische und mathematikdidaktische Wissen angehende Lehrpersonen am Ende ihrer Lehrerausbildung verfügen. Nachdem in Kapitel 10 dieses Bands das mathematische Wissen im Fokus stand, wird in diesem Kapitel das mathematik- 
didaktische Wissen untersucht. Die Analysen sollen Antworten auf die folgenden Fragestellungen liefern:

1. Über welches Wissen in Mathematikdidaktik verfügen die angehenden Mathematiklehrpersonen der Deutschschweiz im internationalen Vergleich?

a) Lassen sich in den mathematikdidaktischen Leistungen der angehenden Lehrpersonen der Deutschschweiz im Vergleich mit anderen Ländern Unterschiede feststellen?

b) Wie gross ist die Streuung der Mathematikleistungen innerhalb der einzelnen Länder?

c) Welcher Anteil der angehenden Mathematiklehrpersonen der Deutschschweiz erreicht hohe Leistungen in der Mathematikdidaktik? Welcher Anteil erreicht unbefriedigende Leistungen?

2. Unterscheiden sich die Leistungen in Mathematikdidaktik zwischen den Lehrerbildungsinstitutionen bzw. den verschiedenen Ausbildungsgängen in der Deutschschweiz?

3. Welche individuellen Merkmale charakterisieren angehende Lehrpersonen mit hohen Leistungen in der Mathematikdidaktik?

a) Wie hängen Geschlecht, soziale Herkunft und Vorbildung mit mathematikdidaktischem Wissen zusammen?

b) Welche Zusammenhänge bestehen zwischen motivationalen Orientierungen und mathematikdidaktischem Wissen?

Der Beitrag ist so aufgebaut, dass zunächst eine Beschreibung der im Rahmen von TEDS-M verwendeten Konzeption zum mathematikdidaktischen Wissen folgt. Anhand von Beispielaufgaben wird erläutert, welche Testhinhalte erfragt wurden. Im Ergebnisteil werden, ausgehend von obigen Fragestellungen, jeweils zuerst die Befunde für die Primarlehrpersonen und danach für die Lehrpersonen der Sekundarstufe I dargestellt. Diese Trennung ist nötig, weil die beiden Tests und als Folge davon die berichteten Skalenwerte nicht direkt zwischen den Stufen vergleichbar sind (vgl. Kapitel 2 in diesem Band).

\subsection{Zur Messung des mathematikdidaktischen Wissens}

\subsubsection{Rahmenkonzeption}

Die Konzeption zur Messung fachdidaktischen Wissens bei TEDS-M ${ }^{3}$ unterscheidet, in Anlehnung an die Arbeiten von Shulman (1987) sowie Fan und

3 Genauere Informationen zur Rahmenkonzeption sind dem Framework von TEDS-M (Tatto et al., 2008) und dem internationalen TEDS-M Bericht (Tatto et al., 2012) zu entnehmen. 
Cheong (2002), zwischen zwei Teilbereichen bzw. Subdimensionen. Der Bereich curriculares und planungsbezogenes Wissen geht dem eigentlichen Unterrichten voraus, der Bereich interaktionsbezogenes Wissen wird dagegen erst in der Interaktion mit der Klasse wirksam (vgl. auch Döhrmann et al., 2010).

Die folgende Tabelle aus dem theoretischen Rahmenkonzept von TEDS-M (Tatto et al., 2008, S. 39) beschreibt genauer, was die beiden Dimensionen Curriculum und Planung sowie Interaktion umfassen.

(1a) Mathematical curricular knowledge

- Establishing appropriate learning goals

- Knowing different assessment formats

- $\quad$ Selecting possible pathways and seeing connections within the curriculum

- Identifying the key ideas in learning programs

- Knowledge of mathematics curriculum

(1b) Knowledge of planning for mathematics teaching and learning (preactive)

- $\quad$ Planning or selecting appropriate activities

- Choosing assessment formats

- Predicting typical students' responses, including misconceptions

- Planning appropriate methods for representing mathematical ideas

- Linking the didactical methods and the instructional designs

- Identifying different approaches for solving mathematical problems

- Planning mathematical lessons

(2) Enacting mathematics for teaching and learning (interactive)

- Analyzing or evaluating students'mathematical solutions or arguments

- Analyzing the content of students'questions

- Diagnosing typical students' responses, including misconceptions

- Explaining or representing mathematical concepts or procedures

- Generating fruitful questions

- $\quad$ Responding to unexpected mathematical issues

- Providing appropriate feedback

Im TEDS-M Test zum mathematikdidaktischen Wissen waren Aufgaben zu allen Teilbereichen enthalten. Die Ergebnisse sind wie diejenigen zum Fachwissen (vgl. Kapitel 10 in diesem Band) zu einer Variablen zusammengefasst und so skaliert, dass der internationale Mittelwert bei 500 liegt und die Standardabweichung 100 Punkte beträgt. Wie beim mathematischen Wissen dürfen aufgrund der Verschiedenheit der Tests auch beim mathematikdidaktischen Wissen die Skalenwerte der angehenden Primarlehrpersonen nicht direkt mit jenen der angehenden Lehrpersonen der Sekundarstufe I verglichen werden. 


\subsubsection{Tests zur Messung mathematikdidaktischen Wissens}

Die Tests zur Messung des mathematikdidaktischen Wissens enthalten etwa gleich viele Aufgaben zu den Teilbereichen curriculares und planungsbezogenes Wissen sowie zum interaktionsbezogenen Wissen. Weil die Tests zur Mathematikdidaktik mit 34 Aufgaben (Primarstufe) bzw. 29 Aufgaben (Sekundarstufe I) weniger Aufgaben aufweisen als jene zum Mathematikwissen, kann zur Beschreibung der erreichten mathematikdidaktischen Leistung nur je ein Referenzwert verwendet werden. Dieser erlaubt eine grobe Einteilung in zwei Leistungsniveaus.

\section{Referenzwert für das mathematikdidaktische Wissen angehender} Primarstufenlehrpersonen

Der Referenzwert für die mathematikdidaktische Leistung angehender Primarstufenlehrpersonen liegt mit 544 Punkten deutlich über dem internationalen Mittelwert von 500 Punkten. Angehende Lehrpersonen im Leistungsbereich von 544 Punkte können z.B. richtig einschätzen, ob eine beschriebene Lehrstrategie für ein gegebenes Beispiel passend ist oder nicht, und sie sind in der Lage, Arbeiten von Lernenden zu bewerten, wenn es sich um einfache und typische Inhalte der Primarstufe handelt. Sie können wie in der Beispielaufgabe (Abbildung 11.1) richtig erkennen, welche arithmetischen Elemente in einstufigen Textaufgaben den Schwierigkeitsgrad für Lernende beeinflussen und mitbestimmen.

Abbildung 11.1:TEDS-M Beispielaufgabe aus dem Bereich Curriculum und Planung

Die Lehrerin einer ersten Klasse fordert ihre Kinder dazu auf, die folgenden vier Sachaufgaben auf ihre eigene Art und Weise zu bearbeiten, gegebenenfalls unter Verwendung von Material, falls gewünscht.

Aufgabe 1: Jana hat 3 Blätter mit Aufklebern. Auf jedem Blatt sind 6 Aufkleber. Wie viele Aufkleber hat Jana insgesamt?

Aufgabe 2: Jürgen hat in seinem Aquarium 5 Fische. Er bekam zu seinem Geburtstag 7 weitere Fische. Wie viele Fische hat Jürgen jetzt?

Aufgabe 3: Jonas hatte einige Spielzeugautos. Er hat 7 davon verloren. Jetzt hat er noch 4 Autos übrig. Wie viele Autos hatte Jonas, bevor er welche verloren hat?

Aufgabe 4: Manuela hatte 13 Luftballons. 5 Ballons sind geplatzt. Wie viele Ballons hat Manuela noch übrig?

Die Lehrerin bemerkt, dass zwei der Aufgaben für ihre Klasse schwieriger zu lösen sind als die anderen beiden.

Geben Sie an, welche ZWEI Aufgaben für Erstklässler wohl SCHWIERIGER zu lösen sind.

Aufgabe und Aufgabe 
Sie verfügen über gewisse Fähigkeiten im Interpretieren von Lösungsmethoden der Lernenden sowie im Erkennen und Benennen von Schülerschwierigkeiten beim Lösen von Aufgaben. Allerdings können sie diese weniger klar und stimmig artikulieren als angehende Lehrpersonen mit höheren Leistungen in der Mathematikdidaktik. In gleicher Weise können sie Eigenschaften grafischer Repräsentationen von Schülerantworten identifizieren und benennen, jedoch nicht so gut wie angehende Lehrpersonen mit höheren Leistungswerten.

Angehende Lehrpersonen mit Leistungen um den Referenzwert sind auch eher nicht in der Lage zu beurteilen, wann eine grafische Darstellung als Anschauungsmittel nicht mehr eingesetzt werden soll, oder zu entscheiden, wie die Denkweise einer Schülerin in einem algebraischen Term repräsentiert ist (vgl. Aufgabe in Abbildung 11.2).

Abbildung 11.2: TEDS-M Beispielaufgabe aus dem Bereich Interaktion

Annette bildet aus Zahnstochern eine Folge von geometrischen Figuren, indem sie dem unten dargestellten Konstruktionsprozess folgt. Jede neue Figur enthält ein weiteres Dreieck.

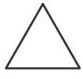

$t=1$

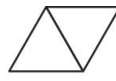

$t=2$

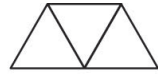

$t=3$

Die Variable t zeigt die Position der Figur innerhalb der Folge an.

Annette erklärt ihre Vorgehensweise zur Entwicklung einer mathematischen Beschreibung des Konstruktionsprozesses wie folgt:

„Zuerst habe ich drei Zahnstocher für jedes Dreieck benutzt."

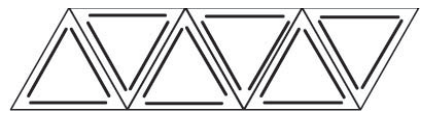

„Aber dann habe ich gesehen, dass ich einen Zahnstocher in jedem Dreieck zweimal gezählt habe, ausser den letzten, also musste ich diese wieder abziehen."

Die Variable $n$ steht für die Anzahl der Zahnstocher, die für eine Figur verwendet werden. Welche der folgenden Gleichungen spiegelt am ehesten Annettes Aussage wider?

Kreuzen Sie bitte ein Feld an.
A. $\quad n=2 t+1$
B.
$\mathrm{n}=2(\mathrm{t}+1)-1$
C.
$\mathrm{n}=3 \mathrm{t}-(\mathrm{t}-1)$
D. $\mathrm{n}=3 \mathrm{t}+1-\mathrm{t}$

$$
\begin{aligned}
& \square_{1} \\
& \square_{2} \\
& \square_{3} \\
& \square_{4}
\end{aligned}
$$


Unter Umständen wissen angehende Lehrpersonen auf dieser Stufe auch nicht, warum ein bestimmtes methodisches Vorgehen sinnvoll ist oder ob sich eine Rechenstrategie auf eine grössere Klasse von Problemen verallgemeinern lässt oder gar Allgemeingültigkeit aufweist.

Sie sind sich gängiger Fehlvorstellungen wie im Beispiel von Abbildung 11.3 eher nicht bewusst oder können, wie in Teilaufgabe b) des Beispiels verlangt, keine passende grafische Darstellung zum fraglichen Zahlzusammenhang erstellen.

\section{Abbildung 11.3: TEDS-M Beispielaufgabe aus dem Bereich Interaktion}

Jeremy stellt fest, dass wenn er 0,2 $\times 6$ in seinen Taschenrechner eintippt, das Ergebnis kleiner ist als 6 , und wenn er $6 \div 0,2$ eingibt, das Ergebnis grösser ist als 6 . Dies irritiert ihn, und er fragt seinen Lehrer nach einem neuen Taschenrechner.

a) Welche Fehlüberlegung hat Jeremy vermutlich gemacht?

b) Zeichnen Sie eine Darstellung, um die Aufgabe 0,2 $\times 6$ für Jeremy zu veranschaulichen, damit dieser nachvollziehen kann, WARUM die Antwort des Taschenrechners so lautet.

Referenzwerte für das mathematikdidaktische Wissen angehender Lehrpersonen der Sekundarstufe I

Für das mathematikdidaktische Wissen angehender Lehrpersonen der Sekundarstufe I konnte aufgrund der relativ geringen Aufgabenanzahl wie für die Primarstufe nur ein Referenzwert etabliert werden. Der Referenzwert unterteilt die Leistungen in zwei unterschiedliche Niveaus und liegt mit 509 Punkten knapp über dem internationalen Durchschnitt.

Angehende Lehrpersonen der Sekundarstufe I mit einer Leistung an diesem Referenzwert verfügen über ein unterschiedliches Spektrum an Wissen bezüglich des Curriculums der Sekundarstufe I und der Planung von Unterrichtslektionen. So wissen sie normalerweise welches Vorwissen für die Herleitung der Lösungsformel für quadratische Gleichungen nötig ist und welche Folgen es hätte, wenn man das Thema Quadratwurzeln aus der Sekundarstufe I in die Sekundarstufe II verlagerte. Hingegen haben sie aber Schwierigkeiten zu entscheiden, welches mathematische Konzept (Kongruenz) beim Beweis einer Aussage zu gleichschenkligen Dreiecken nützlich sein könnte.

Eine Person mit diesem Leistungsniveau verfügt über gewisse Kenntnisse im Bereich der Schulmathematik. Sie kann in manchen Fällen die mathematischen Arbeiten einer Schülerin oder eines Schülers richtig beurteilen. So kann sie z.B. bewerten, ob das Diagramm eines Schülers bestimmte geometrische Bedingungen erfüllt oder nicht, oder sie kann in der in Abbildung 11.4 dargestellten Aufgabe erkennen, dass die in Worten aufgeschrieben Begründung für einen Sachverhalt richtig ist. Weil sie auf diesem Niveau aber über kein gut ausgebildetes Verständnis dazu verfügt, was ein gültiges mathematisches Argument ist und was nicht, hat sie manchmal Schwierigkeiten damit, nicht ausreichende Argumente als solche zu erkennen (z.B. Leons Antwort im Beispiel). 
Abbildung 11.4: TEDS-M Beispielaufgabe aus dem Bereich Interaktion

Einige Schülerinnen und Schüler der Sekundarstufe I wurden aufgefordert, die folgende Aussage zu beweisen:

Wenn man drei aufeinander folgende natürliche Zahlen multipliziert, dann ist das

Ergebnis ein Vielfaches von 6.

Nachfolgend drei Antworten.

\section{Katjas Antwort}

Ein Vielfaches von 6 muss die Teiler 3 und 2 besitzen.

Wenn man 3 aufeinander folgende Zahlen hat, dann ist

eine davon ein Vielfaches von 3.

Ausserdem ist mindestens eine Zahl gerade, und alle geraden Zahlen sind Vielfache von 2.

Wenn man die drei aufeinander folgenden Zahlen multipliziert, muss das Ergebnis mindestens einmal den Teiler 3 und einmal den Teiler 2 besitzen.

$$
\begin{aligned}
& \text { Leons Antwort } \\
& \begin{array}{l}
1 \times 2 \times 3=6 \\
2 \times 3 \times 4=24=6 \times 4 \\
4 \times 5 \times 6=120=6 \times 20 \\
6 \times 7 \times 8=336=6 \times 56
\end{array}
\end{aligned}
$$

$$
\begin{aligned}
& \text { Marias Antwort } \\
& \begin{aligned}
n \times(n+1) \times(n+2)= & \left(n^{2}+n\right) \times(n+2) \\
= & n^{3}+n^{2}+2 n^{2}+2 n
\end{aligned} \\
& \text { Kürzen der n's ergibt } \quad 1+1+2+2=6
\end{aligned}
$$

Entscheiden Sie, ob die Beweise gültig sind.

Kreuzen Sie bitte ein Feld pro Zeile an.

Gültig

Nicht gültig
A. Katjas Beweis
B. Leons Beweis
C. Marias Beweis

$\begin{array}{ll}\square_{1} & \square_{2} \\ \square_{1} & \square_{2} \\ \square_{1} & \square_{2}\end{array}$

Personen auf dieser Leistungsstufe sind eher nicht in der Lage, die richtige Lösung für ein trigonometrisches Problem zu erkennen und können Beispiele von Schülerantworten zu einer das lineare und exponentielle Wachstum betreffenden Aufgabe nicht konsistent auf einer dreistufigen Bewertungsskala einordnen.

Schülerfehler oder Verständnisschwierigkeiten können sie dann erfolgreich identifizieren und analysieren, wenn die Schülerarbeiten einschrittige oder kurze Erklärungen beinhalten. Handelt es sich um komplexere mathematische Situationen, sind sie jedoch weniger erfolgreich. So können sie eher 
nicht erklären, warum in Abbildung 11.5 die zweite der gegebenen Textaufgaben (Aufgabe b) für Lernende häufig schwieriger zu lösen ist. Sie sind ebenso eher nicht in der Lage zu erkennen, ob vorgegebene Textaufgaben einen Term zur Division von Brüchen korrekt repräsentieren.

Abbildung 11.5: TEDS-M Beispielaufgabe zur Erfassung (a) mathematischen Wissens im Bereich Algebra und (b) mathematikdidaktischen Wissens im Bereich Interaktion

Die folgenden Sachaufgaben stammen aus einem Mathematikbuch der Sekundarstufe I.

1. Peter, David und Jonathan spielen mit Murmeln. Zusammen haben sie 198 Murmeln. Peter hat 6-mal so viele Murmeln wie David und Jonathan hat 2-mal so viele Murmeln wie David. Wie viele Murmeln hat jeder der Jungen?

2. Die drei Kinder Anna, Philipp und Lukas besitzen zusammen 198 Schweizer Franken. Anna hat 6-mal so viel Geld wie Philipp und 3-mal so viel wie Lukas. Wie viele Schweizer Franken hat jedes Kind?

(a) Lösen Sie beide Aufgaben.

(b) Üblicherweise bereitet die zweite Aufgabe Schülerinnen und Schülern der Sekundarstufe I grössere Probleme als die erste. Nennen Sie einen Grund, der für den unterschiedlichen Schwierigkeitsgrad verantwortlich sein könnte.

\subsection{Mathematikdidaktisches Wissen angehender Primarlehrpersonen}

\subsubsection{Mathematikdidaktisches Wissen im Ländervergleich}

International sind die Unterschiede im mathematikdidaktischen Wissen der angehenden Primarlehrpersonen sehr gross. Die Differenz zwischen dem niedrigsten Mittelwert (Georgien) und dem höchsten (Singapur) beträgt fast 248 Punkte.

Das höchste mathematikdidaktische Wissen weisen die angehenden Primarlehrpersonen der beiden ostasiatischen Länder Taiwan und Singapur aus (Abbildung 1.1). Die Leistungen in diesen beiden Ländern sind mit einem Abstand von 55 bzw. 56 Punkten signifikant besser als in der Deutschschweiz, welche einen Mittelwert von 537 Punkten erreicht. Die USA unterscheidet sich statistisch nicht von der Deutschschweiz. Die übrigen Länder liegen signifikant hinter den Ergebnissen der Deutschschweiz zurück, darunter auch die europäischen Länder Deutschland, Spanien und Polen. Die Leistungen der Deutschschweiz in der Mathematikdidaktik sind signifikant höher als im internationalen Durchschnitt aller an TEDS-M beteiligten Länder.

Obschon die Streuung in der Deutschschweiz mit einer Standardabweichung von 64 Punkten vergleichsweise niedrig ist, sind die Leistungsunter- 
schiede zwischen den künftigen Lehrpersonen in der Deutschschweiz beachtlich. So beträgt in der Mathematikdidaktik der Mindestabstand zwischen den $25 \%$ schwächsten und den $25 \%$ besten angehenden Primarlehrpersonen 76 Punkte. Dieser ist damit deutlich grösser als der durchschnittliche Rückstand der Deutschschweiz auf die beiden führenden Länder Singapur und Taiwan. Zudem zeigt sich, dass in den beiden ostasiatischen Ländern Taiwan und Singapur sowie in der Schweiz jeweils mindestens $75 \%$ der angehenden Lehrpersonen über dem internationalen Mittelwert liegen, was auf ein nahezu flächendeckendes hohes Wissensniveau schliessen lässt.

Während in der Deutschschweiz die besten 5\% der angehenden Primarlehrpersonen Leistungen von mindestens 629 Punkten erreichen, liegen die Spitzenwerte in Singapur und Taiwan bei über 700 Punkten. Weniger gross ist der Abstand mit 57 bzw. 51 Punkten gegenüber den Spitzenländern am unteren Ende der Skala der Mathematikdidaktik. In der Deutschschweiz erzielen 5\% weniger als 433 Punkte. Länder wie Georgien, Polen oder auch Deutschland weisen eine markant grössere Leistungsbandbreite auf als die Deutschschweiz. Entsprechend niedrig sind demzufolge in diesen Ländern die schwächsten Leistungen in der Mathematikdidaktik.

Abbildung 11.6: Mathematikdidaktisches Wissen von angehenden Primarlehrpersonen im Ländervergleich

$\begin{array}{lccc}\text { Länder } & \text { M } & \text { SE } & \text { SD } \\ \text { Singapur } & 593 & (3.4) & 71 \\ \text { Taiwan } & 592 & (2.3) & 68 \\ { }^{1} \text { USA } & 544 & (2.5) & 68 \\ \text { Deutschschweiz } & 537 & (1.6) & 64 \\ \text { Russland } & 512 & (8.1) & 83 \\ \text { Thailand } & 506 & (2.3) & 70 \\ \text { Malaysia } & 503 & (3.1) & 67 \\ \text { Deutschland } & 502 & (4.0) & 92 \\ \text { Spanien } & 492 & (2.2) & 63 \\ { }^{1} \text { Polen } & 478 & (1.8) & 101 \\ \text { Philippinen } & 457 & (9.7) & 67 \\ \text { Botswana } & 448 & (8.8) & 75 \\ { }^{1} \text { Chile } & 425 & (3.7) & 90 \\ \text { Georgien } & 345 & (4.9) & 100\end{array}$

Mittelwert sig. höher als in der Deutschschweiz Mittelwert nicht sig. vers chieden von der Deutschschweiz Mittelwert sig. tiefer als in der Deutschschweiz

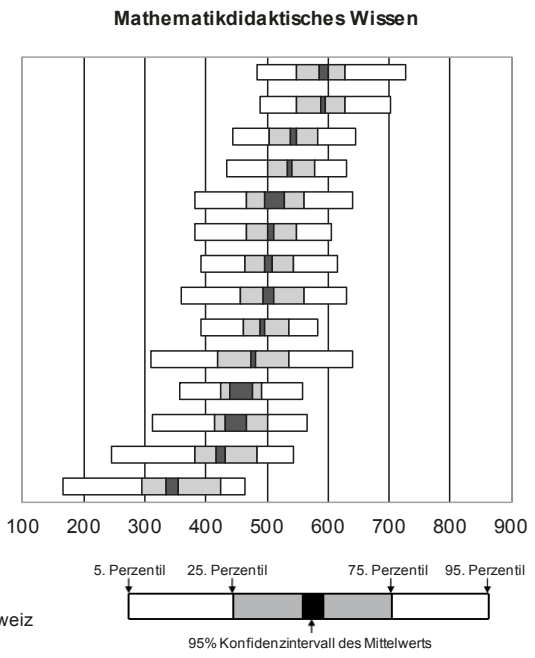

Anmerkungen: $\mathrm{M}=$ Mittelwert; $\mathrm{SE}=$ Standardfehler des Mittelwerts; $\mathrm{SD}=$ Standardabweichung. Die Testleistungen sind so skaliert, dass der internationale Mittelwert 500 Punkte und die Standardabweichung 100 Punkte betragen.

1) USA, Polen und Chile weisen eine Rücklaufquote von weniger als $75 \%$ auf. 


\subsubsection{Mathematikdidaktisches Wissen nach Ausbildungsgängen im internationalen Vergleich}

Die bisher dargestellten Ländervergleiche legten dar, über welches mathematikdidaktische Wissen angehende Lehrpersonen mit erworbener Lehrberechtigung auf der Primarstufe verfügen. Dabei wurde nicht zwischen verschiedenen Ausbildungsgängen unterschieden, etwa in Bezug auf den Spezialisierungsgrad der Ausbildung oder den Erwerb der Lehrberechtigung für eine begrenzte Anzahl Jahrgangsstufen. Um den unterschiedlichen Ausbildungsmerkmalen in den verschiedenen Ländern gerecht zu werden, stehen bei den nachfolgenden Darstellungen die Ergebnisse der unterschiedlichen Ausbildungsgänge im Fokus (vgl. Kapitel 2 in diesem Band). Eine Unterscheidung betrifft die Ausbildung zur Lehrperson der Unteren Primarstufe (1-4) bzw. zur Primarlehrperson (1-6). Angehende Lehrpersonen der Unteren Primarstufe (1-4) erlangen eine Lehrberechtigung bis maximal zur vierten Primarklasse, angehende Primarlehrpersonen (1-6) dürfen bis zur sechsten Klassenstufe unterrichten. Die Ausbildung zur Primarlehrperson kann weiter in Generalisten- und SpezialistenAusbildungen unterteilt werden. Letztere erhalten die Lehrberechtigung nur für eine begrenzte Fächeranzahl.

Abbildung 11.7: Mathematikdidaktisches Wissen angehender Primarlehrpersonen nach Ausbildungstypen im internationalen Vergleich

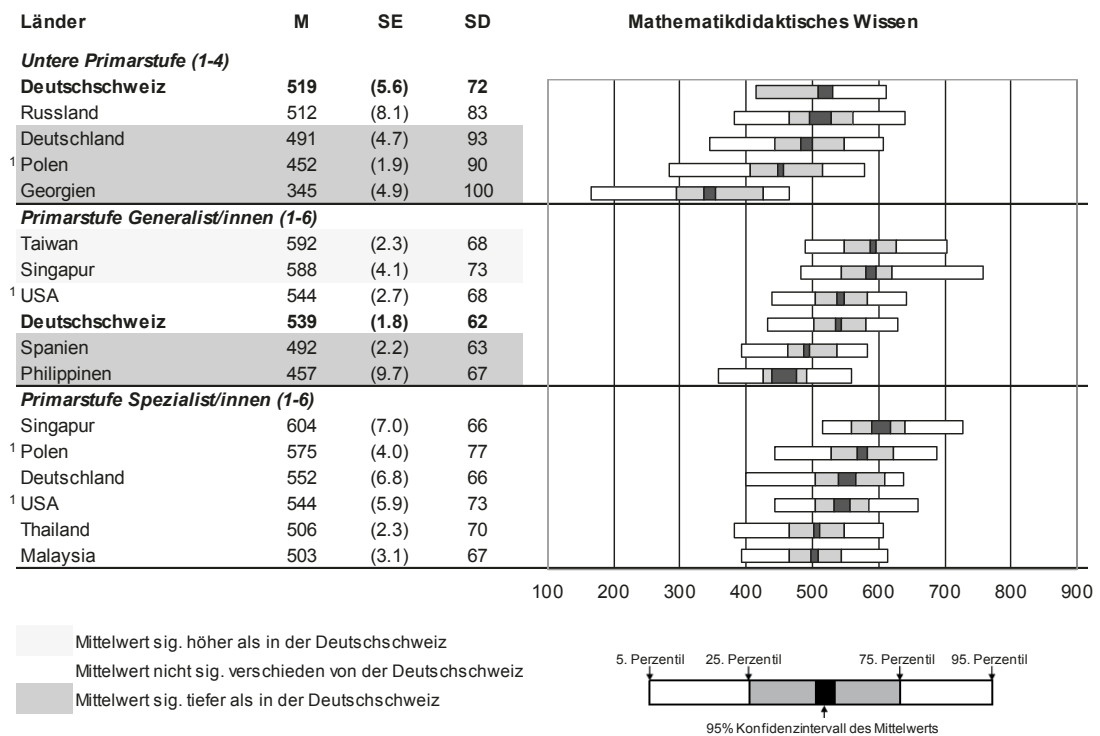

Anmerkungen: $\mathrm{M}=$ Mittelwert; $\mathrm{SE}=$ Standardfehler des Mittelwerts; $\mathrm{SD}=$ Standardabweichung. 1) USA und Polen weisen eine Rücklaufquote von weniger als $75 \%$ auf. 
Vergleicht man die Länderergebnisse zum mathematikdidaktischen Wissen nach den verschiedenen Ausbildungstypen, so steht die Deutschschweiz auf der Unteren Primarstufe (1-4) gemeinsam mit Russland an der Spitze (Abbildung 11.7). Die Leistungen angehender Lehrpersonen der Unteren Primarstufe (1-4) in Deutschland sind, anders als beim Mathematikwissen (vgl. Kapitel 10 in diesem Band), signifikant tiefer. In der Gruppe der Generalistenausbildungen (1-6) befindet sich die Deutschschweiz zusammen mit den USA im Mittelfeld. Die asiatischen Länder heben sich signifikant von den Leistungen der Deutschschweiz ab, hingegen weisen Spanien und die Philippinen deutlich niedrigere Leistungen auf als die Deutschschweiz.

Die Schweiz bildet im Bereich der Primarstufe keine Spezialistinnen und Spezialisten aus. Die als Fachspezialistinnen und -spezialisten ausgebildeten Lehrpersonen in Deutschland unterscheiden sich in ihren Leistungen in der Mathematikdidaktik jedoch nicht signifikant von den Generalistinnen und Generalisten in der Deutschschweiz.

Innerhalb der Deutschschweiz zeigen sich Unterschiede in der mathematikdidaktischen Leistung zwischen den Ausbildungsgängen. Das mathematikdidaktische Wissen der angehenden Lehrpersonen für die Untere Primarstufe (1-4) liegt in der Deutschschweiz mit 519 Punkten um 20 Punkte unter jenem der künftigen Lehrpersonen mit Lehrberechtigung für die Primarstufe (1-6). Dieser Unterschied zwischen den beiden Zielstufen ist zwar statistisch signifikant $(\mathrm{t}=3.34 ; \mathrm{p}<.001)$, aber mit einer Effektstärke von $\mathrm{d}=0.31$ als eher gering zu bewerten. Die Differenz geht insbesondere auf Unterschiede bei den schwächsten Leistungen zurück. Die Leistungen in Mathematikdidaktik sind bei den schwächsten 5\% der angehenden Unterstufenlehrpersonen um 18 Punkte niedriger als bei den künftigen Lehrpersonen mit Lehrberechtigung für die ganze Primarstufe.

Wie es zu diesem Unterschied im Bereich des mathematikdidaktischen Wissens kommt, ist noch ungeklärt. Der Unterschied ist dann weniger problematisch, wenn er von Testaufgaben herrührt, die inhaltlich über dem Niveau der Unterstufe liegen. Dem ist in weiterführenden Analysen noch genauer nachzugehen. Da mathematisches Wissen eng mit fachdidaktischem Wissen zusammenhängt (Baumert \& Kunter, 2011), dürfte dies zumindest teilweise erklären, dass sich bei der Fachdidaktik ein ähnlicher Leistungsunterschied zwischen den angehenden Unterstufen- und Primarlehrpersonen zeigt wie beim mathematischen Wissen.

\section{Mathematikdidaktisches Wissen nach Leistungsniveau}

Die mathematikdidaktischen Leistungen der angehenden Primarlehrpersonen lassen sich einem von zwei Leistungsniveaus zuordnen. Der Referenzwert, der die beiden Gruppen teilt, liegt bei 509 Punkten (zur inhaltlichen Beschreibung von Leistungen um den Referenzwert vgl. Kapitel 11.2.2). 
Über alle Länder hinweg weisen ca. 30\% der angehenden Primarlehrpersonen ein hohes Leistungsniveau in Mathematikdidaktik auf. Betrachtet man die Leistungsniveaus in Mathematikdidaktik nach Ausbildungstypen so verfügen international die Spezialistinnen und Spezialisten der Primarstufe (1-6) klar über die höchsten Anteile im oberen Leistungsniveau (Abbildung 11.8). Bei den angehenden Lehrpersonen der Unteren Primarstufe (1-4) erreicht generell ein deutlich geringerer Anteil das höhere Leistungsniveau als im Ausbildungstyp Primarstufe (1-6). Dies gilt auch für die Deutschschweiz, wo bei den angehenden Primarlehrpersonen der Unteren Primarstufe (1-4) eine deutlich grössere Gruppe nur das niedrigere Leistungsniveau (68\%) erreicht als bei der Primarstufe (1-6;56\%). Umgekehrt formuliert erreichen nur 32\% (Primarstufe 1-4) bzw. 44\% (Primarstufe 1-6) das höhere Leistungsniveau in der Mathematikdidaktik.

Die Deutschschweiz liegt bei der Primarstufe (1-4) in etwa gleichauf mit Russland und Deutschland, wohingegen Polen und Georgien über deutlich weniger angehende Lehrpersonen auf dem höheren Leistungsniveau verfügen. Bei der Primarstufe (1-6) befindet sich die Deutschschweiz mit 44\% angehender Lehrpersonen, welche das höhere Leistungsniveau erreichen, im Mittelfeld.

Abbildung 11.8: Mathematikdidaktisches Wissen von angehenden

Primarlehrpersonen nach Leistungsniveau

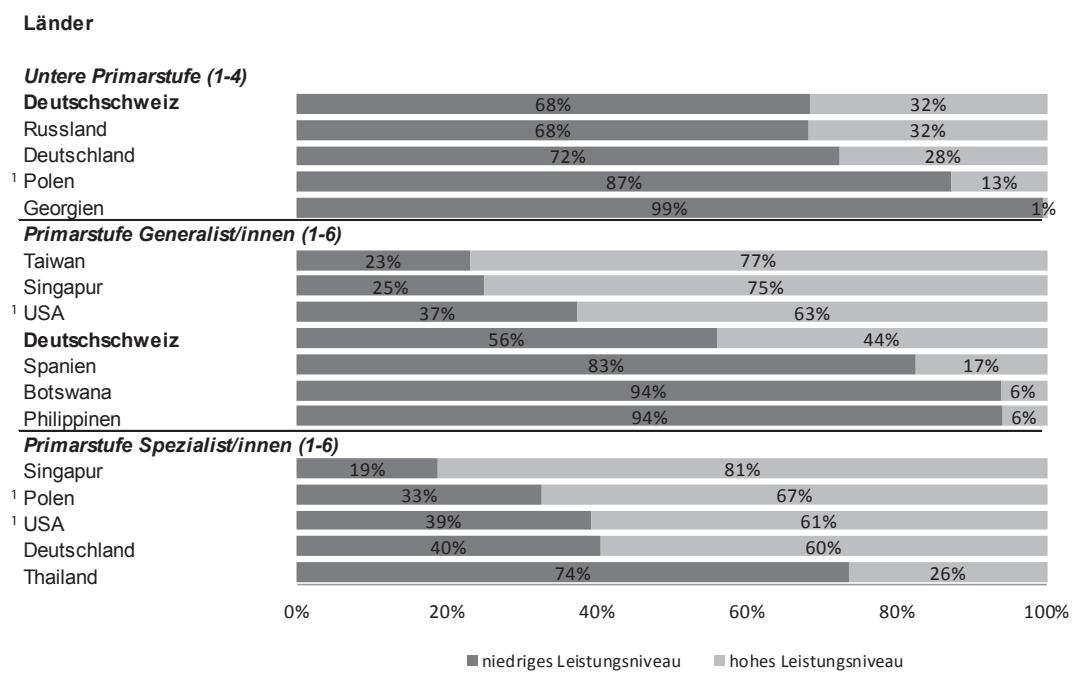

Anmerkungen: ${ }^{1)}$ USA und Polen weisen eine Rücklaufquote von weniger als $75 \%$ auf. 


\subsubsection{Mathematikdidaktisches Wissen nach Ausbildungsgängen in der Deutschschweiz}

Aus Abbildung 11.9 sind die Mittelwerte in der Mathematikdidaktik der einzelnen Lehrerbildungsinstitutionen der Deutschschweiz, aufgeteilt nach Ausbildungsgängen, zu entnehmen. Mit einem „P“ gekennzeichnete Ausbildungsgänge bilden Generalistinnen und Generalisten der Primarstufe (1-6) aus, mit „U“ gekennzeichnete Ausbildungsgänge Lehrpersonen der Unteren Primarstufe (1-4).

Abbildung 11.9: Mathematikdidaktisches Wissen von angehenden

Primarlehrpersonen nach Institutionen in der Deutschschweiz

\begin{tabular}{|c|c|c|c|}
\hline Ausbildungsgänge & M & SE & SD \\
\hline \multicolumn{4}{|c|}{ Untere Primarstufe (1-4) } \\
\hline U1 & 554 & $(13.0)$ & 57 \\
\hline U3 & 553 & (16.1) & 46 \\
\hline U2 & 522 & $(21.4)$ & 47 \\
\hline U6 & 521 & $(19.0)$ & 66 \\
\hline Deutschschweiz & 519 & $(5.6)$ & 72 \\
\hline U5 & 513 & $(9.2)$ & 60 \\
\hline U4 & 507 & $(14.7)$ & 69 \\
\hline \multicolumn{4}{|l|}{ Primarstufe (1-6) } \\
\hline P9 & 589 & $(18.4)$ & 55 \\
\hline P14 & 579 & $(16.4)$ & 78 \\
\hline P7 & 553 & $(12.5)$ & 62 \\
\hline P8 & 552 & $(7.8)$ & 37 \\
\hline P5 & 551 & $(6.8)$ & 59 \\
\hline P11 & 546 & $(11.1)$ & 74 \\
\hline P16 & 546 & $(6.4)$ & 55 \\
\hline $\mathrm{P} 13$ & 540 & $(8.0)$ & 56 \\
\hline $\mathrm{P} 4$ & 540 & $(4.7)$ & 64 \\
\hline Deutschschweiz & 539 & (1.8) & 62 \\
\hline P6 & 536 & (7.0) & 53 \\
\hline P10 & 532 & $(9.0)$ & 34 \\
\hline P12 & 532 & (10.3) & 79 \\
\hline P1 & 530 & (6.3) & 56 \\
\hline P2 & 528 & (11.7) & 40 \\
\hline P3 & 527 & $(6.0)$ & 55 \\
\hline P15 & 508 & (7.6) & 58 \\
\hline
\end{tabular}

Mathematikdidaktisches Wissen angehender

Primarlehrpersonen der Deutschschweiz nach Ausbildungsgängen

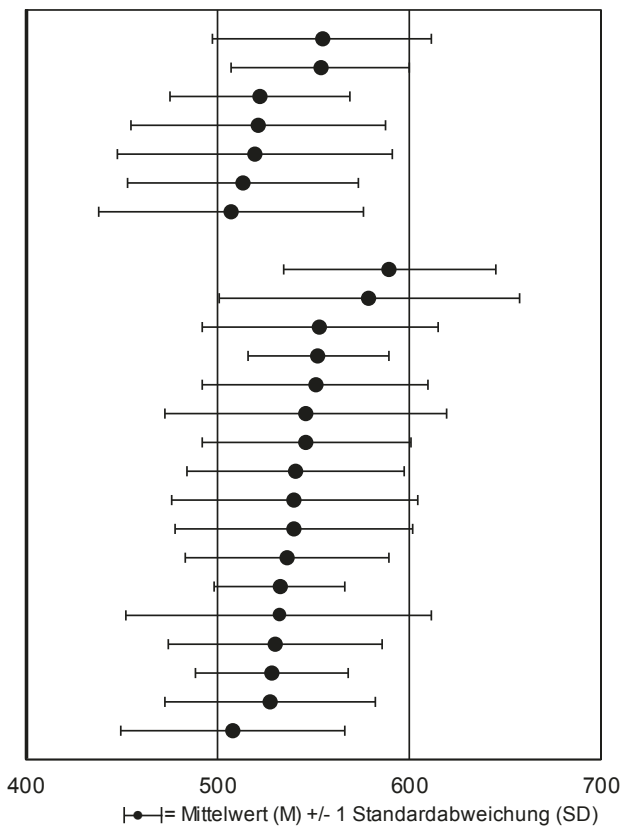

Mittelwert sig. höher als in der Deutschschweiz

Mittelwert nicht sig. verschieden von der Deutschschweiz

Mittelwert sig. tiefer als in der Deutschschweiz

Anmerkungen: $\mathrm{M}=$ Mittelwert; $\mathrm{SE}=$ Standardfehler des Mittelwerts; $\mathrm{SD}=$ Standardabweichung.

Vergleicht man die verschiedenen Lehrerbildungsinstitutionen bzw. Ausbildungsgänge in der Deutschschweiz miteinander, zeigt sich insgesamt ein Unterschied von 82 Punkten zwischen dem Ausbildungsgang mit den höchsten 
(P9) und den niedrigsten Mathematikleistungen (U4). Innerhalb der Schweiz sind also Ausbildungsgänge zu finden, deren Abgängerinnen und Abgänger im Durchschnitt gleich gute Leistungen in Mathematikdidaktik erbringen wie durchschnittliche Lehrpersonen bei Ausbildungsende in Taiwan oder Singapur. Anders herum gibt es Ausbildungsgänge, deren Absolventinnen und Absolventen nur etwa ein mittleres Leistungsniveau thailändischer Lehrpersonen erreichen. Auf der Primarstufe (1-6) fällt eine Institution (P15) leistungsmässig signifikant vom deutschschweizerischen Mittelwert für den Ausbildungstyp ab. Die Streuung der einzelnen Institutionen zeigen teilweise grosse Unterschiede. Es gibt Institutionen mit relativ homogenen Leistungen (v.a. P10, P8, P2), andere Institutionen weisen sehr heterogene Leistungen (v.a. P12, P14, P11) ihrer Abgängerinnen und Abgänger auf. Die Unterschiede sind jedoch geringer als beim mathematischen Wissen (vgl. Kapitel 10 in diesem Band).

\subsubsection{Individuelle Merkmale und mathematikdidaktisches Wissen}

Geschlechterunterschiede im mathematikdidaktischen Wissen

Anders als beim Fachwissen Mathematik (vgl. Kapitel 10 in diesem Band) besteht bezüglich Mathematikdidaktik kein signifikanter Geschlechterunterschied bei den angehenden Primarlehrpersonen (1-6) in der Deutschschweiz (Abbildung 11.10). Sowohl angehende Lehrerinnen (540 Punkte) als auch angehende Lehrer (536 Punkte) erreichen im Durchschnitt ähnliche Leistungen in der Mathematikdidaktik.

Auch international ist zu erkennen, dass die Geschlechterunterschiede in der Mathematikdidaktik generell geringer ausfallen als in der Mathematik. In den meisten Ländern sind die Differenzen zwischen der mittleren Leistung der Männer und derjenigen der Frauen kleiner als 20 Punkte. Nur in den drei Ländern Polen (Untere Primarstufe, 1-4), Spanien (Primarstufe Generalist/innen, 1-6) und Singapur (Primarstufe Spezialist/innen, 1-6) schneiden die Männer signifikant besser ab als die Frauen. In Malaysia erzielen die Frauen einen signifikant höheren Mittelwert für das mathematikdidaktische Wissen als die Männer. Mit einer Differenz von 12 Punkten und einer Effektstärke von d $=.18$ ist dieser Unterschied aber als sehr gering zu bewerten. In Singapur erreichen dagegen die Männer signifikant höhere Leistungen in der Mathematikdidaktik als die Frauen. Die Geschlechterunterschiede in Deutschland und in der Deutschschweiz sind statistisch nicht signifikant. 
Abbildung 11.10: Geschlechterunterschiede im mathematikdidaktischen Wissen von angehenden Primarlehrpersonen im internationalen Vergleich

\begin{tabular}{lccccc} 
Länder & Frauenanteil & \multicolumn{2}{c}{ Frauen } & \multicolumn{2}{c}{ Männer } \\
& & M & SE & M & SE \\
Untere Primarstufe (1-4) & & & & & \\
Deutschland & $93 \%$ & 492 & $(4.9)$ & 476 & $(15.6)$ \\
Russland & $94 \%$ & 512 & $(8.2)$ & 514 & $(14.1)$ \\
${ }^{1}$ Polen & $98 \%$ & 451 & $(1.9)$ & 479 & $(1.9)$ \\
Deutschschweiz & $\mathbf{9 6 \%}$ & $\mathbf{5 1 7}$ & $\mathbf{( 5 . 4 )}$ & & \\
\hline Primarstufe Generalist/innen (1-6) & & & & \\
Deutschschweiz & $\mathbf{8 3 \%}$ & $\mathbf{5 4 0}$ & $\mathbf{( 2 . 0 )}$ & $\mathbf{5 3 6}$ & $\mathbf{( 5 . 2 )}$ \\
Taiwan & $72 \%$ & 593 & $(2.5)$ & 591 & $(3.9)$ \\
Singapur & $76 \%$ & 587 & $(4.5)$ & 591 & $(9.1)$ \\
Philippinen & $81 \%$ & 456 & $(11.8)$ & 463 & $(5.9)$ \\
${ }^{1}$ USA & $92 \%$ & 543 & $(2.9)$ & 553 & $(6.4)$ \\
Spanien & $81 \%$ & 489 & $(2.3)$ & 506 & $(4.0)$ \\
\hline Primarstufe Spezialist/innen (1-6) & & & & \\
Malaysia & $63 \%$ & 508 & $(4.2)$ & 496 & $(3.9)$ \\
${ }^{1}$ USA & $90 \%$ & 544 & $(5.1)$ & 553 & $(28.0)$ \\
Deutschland & $82 \%$ & 552 & $(8.6)$ & 558 & $(6.1)$ \\
${ }^{1}$ Polen & $78 \%$ & 573 & $(4.6)$ & 583 & $(9.5)$ \\
Singapur & $70 \%$ & 599 & $(5.4)$ & 614 & $(15.4)$ \\
Thailand & $75 \%$ & 502 & $(2.8)$ & 518 & $(5.6)$
\end{tabular}

Geschlechterdifferenz in der Mathematikdidaktik zugunsten der Männer

Geschlechterunterschied nicht signifikant

Geschlechterunterschied signifikant

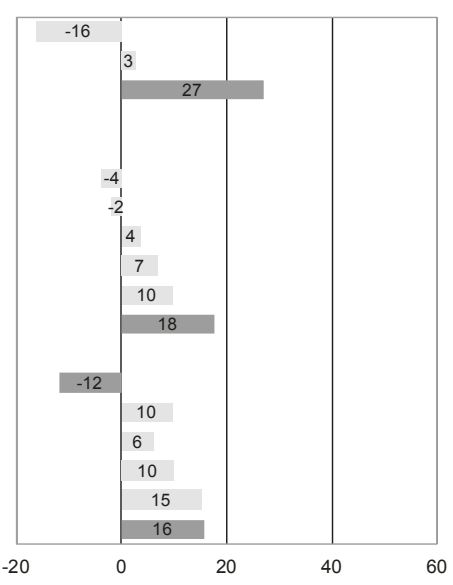

Anmerkungen: $\mathrm{M}=$ Mittelwert; $\mathrm{SE}=$ Standardfehler des Mittelwerts; $\mathrm{SD}=$ Standardabweichung. Die Länder sind innerhalb der Ausbildungstypen nach Grösse des Geschlechterunterschieds sortiert. Georgien ist nicht aufgeführt, weil ausschliesslich Frauen in der Stichprobe waren. Auch für die Deutschschweiz (Untere Primarstufe, 1-4) sind nur die Werte der Frauen dargestellt, da der Männeranteil zu gering ist. Es werden nur diejenigen Ausbildungstypen in der Grafik angezeigt, in denen die Deutschschweiz oder Deutschland enthalten sind.

1) USA und Polen weisen eine Rücklaufquote von weniger als $75 \%$ auf.

\section{Bedeutung individueller Merkmale für das mathematikdidaktische Wissen}

Neben dem Geschlecht wird im Rahmen von TEDS-M auch der Frage nach den Beziehungen zwischen weiteren individuellen Merkmalen und dem mathematikdidaktischen Wissen nachgegangen. Aus den Antworten zum Bildungsniveau der Eltern und der Anzahl Bücher zu Hause wurde ein Index „sozioökonomischer Hintergrund“ gebildet. Als grobe Abschätzung der schulischen Leistungen in der Volksschulzeit wurden die Studierenden gefragt, wie gut ihre Schulnoten im Vergleich mit anderen Schülerinnen und Schülern des Jahrgangs in der Sekundarstufe waren. Dabei wurden die folgenden Antwortmöglichkeiten vorgegeben: 1 = ,normalerweise unter dem Durchschnitt“, 2 = „,normalerweise ungefähr im Durchschnitt“, $3=$,normalerweise besser als der Durchschnitt“, 4 = ,normalerweise unter den Besten“, 5 = ,immer die/der Beste“. 
Abbildung 11.11 bildet das Ergebnis je einer Regression für die Untere Primarstufe (1-4) und die Primarstufe (1-6) ab, aus welcher die Zusammenhänge zwischen dem Geschlecht, dem sozioökonomischen Hintergrund und der Leistungseinschätzung in der eigenen Schulzeit (Sekundarstufe I) zu entnehmen sind. Die Balken zeigen jeweils den Zusammenhang eines Merkmals mit dem mathematikdidaktischen Wissen bei gleichzeitiger Konstanthaltung der anderen Variablen. Statistisch signifikante Zusammenhänge sind dunkel eingefärbt.

Dem ersten Balken in der unteren Abbildung (Primarstufe 1-6) ist die Geschlechterdifferenz im mathematikdidaktischen Wissen zu entnehmen, wobei ein negativer Wert eine höhere Leistung in Mathematikdidaktik zugunsten der Frauen anzeigt. Diese fällt sehr gering aus und liegt im statistischen Zufallsbereich. Der zweite Balken gibt die Leistungsdifferenz an, wenn der sozioökonomische Hintergrund um eine Standardabweichung höher ausfällt. Der dritte Balken verweist auf den Unterschied in der mathematikdidaktischen Leistung bei einer um eine Standardabweichung höheren Leistungseinschätzung.

Die Ergebnisse verdeutlichen, dass das mathematikdidaktische Wissen weder für die Untere Primarstufe (1-4) noch für die Primarstufe (1-6) signifikant mit dem sozioökonomischen Hintergrund zusammenhängt. Die Zusammenhänge zwischen der individuellen Leistungseinschätzung und der mathematikdidaktischen Leistung fallen dagegen für beide Ausbildungstypen signifikant aus. Angehende Primarlehrpersonen, die angeben in der eigenen Sekundarschulzeit bessere Schulnoten als andere erhalten zu haben, schneiden am Ende der Lehrerausbildung im Test zur Mathematikdidaktik besser ab als solche mit einer niedrigeren Leistungseinschätzung.

Abbildung 11.11: Vorhersage mathematikdidaktischen Wissens von angehenden Primarlehrpersonen durch individuelle Merkmale

Untere Primarstufe (1-4)

Sozioökonomischer Hintergrund

Leistungseinschätzung

Primarstufe (1-6)

Geschlecht (männlich)

Sozioökonomischer Hintergrund Leistungseinschätzung
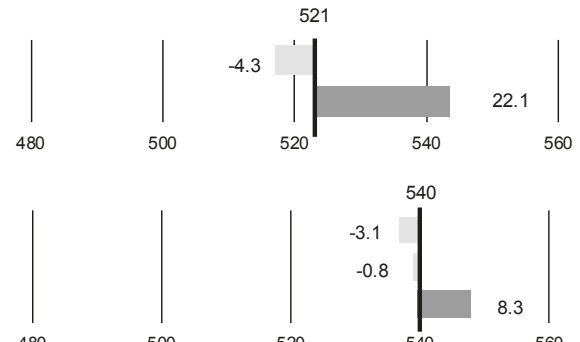

500

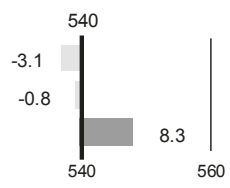

Zusammenhang nicht signifikant

Zusammenhang signifikant

Anmerkung: Die Analysen erfolgten mittels multipler linearer Regression. Das Geschlecht ist dummycodiert (Referenzgruppe "weiblich"); die übrigen Prädiktoren sind z-standardisiert. Die Balkenlängen entsprechen den unstandardisierten Regressionskoeffizienten (B). Aufgrund des geringen Männeranteils in den Ausbildungsgängen der Unteren Primarstufe (1-4) kann zu diesem Ausbildungstyp kein aussagekräftiger Geschlechtervergleich berechnet werden. 


\subsubsection{Motivationale Orientierung und mathematikdidaktisches Wissen}

Zur Untersuchung der Frage nach den Zusammenhängen zwischen den motivationalen Orientierungen und den Leistungen in der Mathematikdidaktik werden die vier Skalen zur Berufsmotivation (fachbezogene Motivation, extrinsische Motivation, Interesse an Lernenden und Interesse an Vermittlung) verwendet, die im Rahmen der Deutschschweizer Zusatzerhebungen zu TEDS-M erfasst wurden (zur Erfassung und Beschreibung der Skalen zur Berufsmotivation vgl. Kapitel 4 in diesem Band).

Die Ergebnisse der multiplen Regression mit den vier unterschiedlichen motivationalen Orientierungen als Prädiktoren des mathematikdidaktischen Wissens ergeben für die angehenden Primarlehrpersonen, dass einzig die fachbezogene Motivation positiv mit der Mathematikdidaktik zusammenhängt $(\mathrm{B}=.14$; Abbildung 11.12). Wer die Auseinandersetzung mit Mathematik als einen wichtigen Grund für die Berufswahl angibt, verfügt am Ende der Ausbildung über ein grösseres mathematikdidaktisches Wissen als weniger Mathematikinteressierte. Die extrinsische Motivation sowie ein höheres pädagogisches Interesse im Bereich der Vermittlung haben einen geringen, aber signifikanten negativen Zusammenhang mit der Leistung in der Mathematikdidaktik. Zwischen dem Interesse an den Lernenden als Berufswahlmotiv und dem mathematikdidaktischen Wissen besteht kein statistisch signifikanter Zusammenhang. Insgesamt zeigt sich also, dass die Beziehungen zwischen Berufsmotivation und mathematikdidaktischem Wissen sehr ähnlich ausfallen wie zuvor zwischen der Berufsmotivation und dem mathematischen Wissen (vgl. Kapitel 10 in diesem Band).

Abbildung 11.12: Zusammenhänge zwischen motivationalen Orientierungen und mathematikdidaktischem Wissen bei angehenden

Primarlehrpersonen in der Deutschschweiz

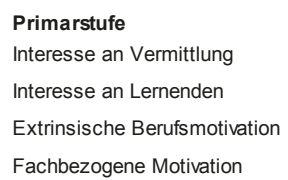

Primarstufe

Interesse an Lernenden

Fachbezogene Motivation

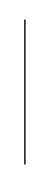

480

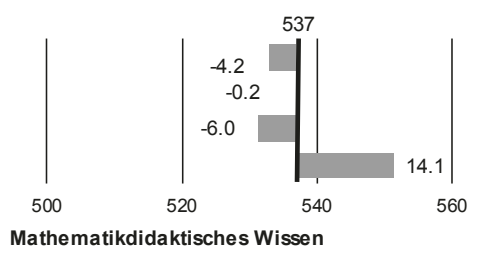

Zusammenhang nicht signifikant

Zusammenhang signifikant

Anmerkung: Die Analysen erfolgten mittels multipler linearer Regression. Die Balkenlängen entsprechen den unstandardisierten Regressionskoeffizienten (B). Alle Prädiktoren sind z-standardisiert. Da sich die Ergebnisse zwischen den beiden Ausbildungstypen "Untere Primarstufe (1-4)" und "Primarstufe (1-6)" nur unwesentlich unterscheiden, sind die Ergebnisse beider Ausbildungstypen gemeinsam dargestellt. 


\subsection{Mathematikdidaktisches Wissen angehender Lehrpersonen der Sekundarstufe I}

\subsubsection{Mathematikdidaktisches Wissen im Ländervergleich}

International sind auch bezüglich des mathematikdidaktischen Wissens der angehenden Lehrpersonen für die Sekundarstufe I grosse Unterschiede festzustellen, auch wenn die Differenz der Mittelwerte zwischen Taiwan und Chile mit 255 Punkten rund 60 Punkte geringer ausfällt als beim mathematischen Wissen. Die durchschnittliche Leistung in der Deutschschweiz liegt mit 549 Punkten auf der Mathematikdidaktik-Skala im ähnlichen Bereich wie die Leistungen der drei Länder Russland, Singapur und Deutschland (Abbildung 11.13). Einzig Taiwan hebt sich markant von allen anderen Ländern ab und erreicht mit einem Vorsprung von 100 Punkten einen signifikant höheren Mittelwert als die Deutschschweiz. Alle anderen Länder erbringen signifikant tiefere Leistungen in der Mathematikdidaktik als die Deutschschweiz.

Die Leistungsstreuung ist in der Deutschschweiz $(\mathrm{SD}=72)$ zwar geringer als in den anderen Spitzenländern, aber doch etwas grösser als beim mathematischen Wissen. Die besten 5\% der angehenden Lehrpersonen erreichen mindestens 671 Punkte, die leistungsschwächsten 5\% weniger als 442 Punkte. Am deutlich längeren Perzentilbalken (Abstand zwischen dem 5. und 95. Perzentil) von Deutschland ist abzulesen, dass dort - wie schon beim mathematischen Wissen - mehr angehende Lehrpersonen eine hohe Leistung im mathematikdidaktischen Test erzielen, aber auch der Anteil von schwachen Leistungen grösser ist als in der Deutschschweiz. 
Abbildung 11.13: Mathematikdidaktisches Wissen von angehenden Lehrpersonen der Sekundarstufe I im Ländervergleich

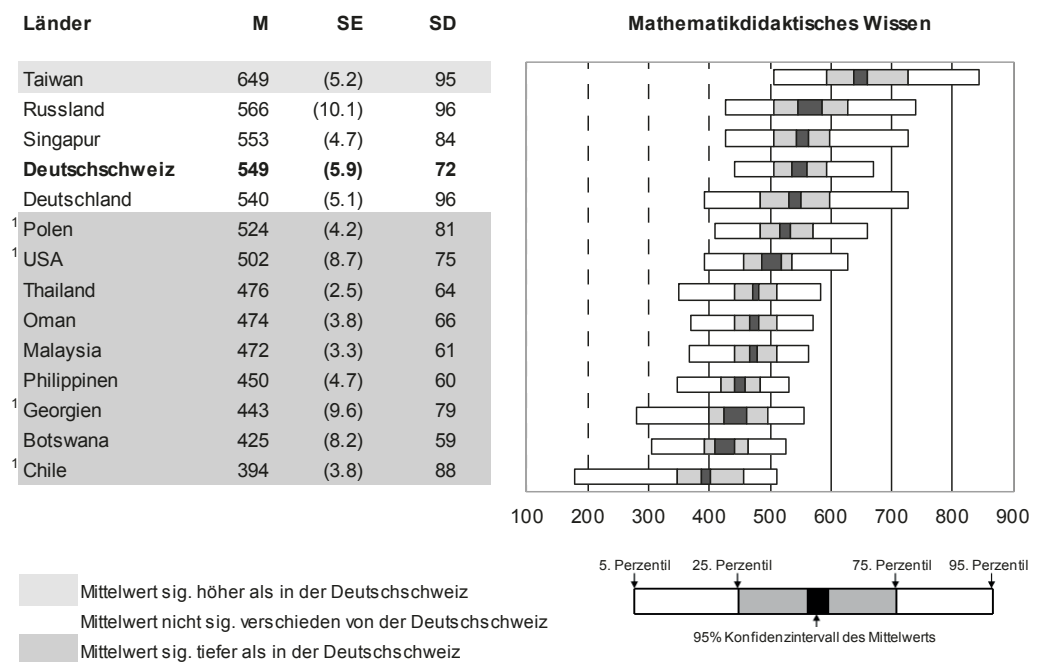

Anmerkungen: $\mathrm{M}=$ Mittelwert; $\mathrm{SE}=$ Standardfehler des Mittelwerts; $\mathrm{SD}=$ Standardabweichung. 1) USA, Polen, Georgien und Chile weisen eine Rücklaufquote von weniger als $75 \%$ auf.

\subsubsection{Mathematikdidaktisches Wissen nach Ausbildungsgängen im internationalen Vergleich}

Betrachtet man die Leistungen in Mathematikdidaktik für die angehenden Lehrpersonen der Sekundarstufe I, aufgeteilt nach den unterschiedlichen Ausbildungstypen, so zeigt sich, dass die Schweiz mit 549 Punkten mit Singapur den Spitzenplatz einnimmt und signifikant höhere Werte in den mathematikdidaktischen Leistungen erzielt als die anderen Länder. Im Ausbildungstyp für die Sekundarstufe I liegt die Differenz zwischen dem niedrigsten (Chile) und dem höchsten Mittelwert (Deutschschweiz) bei 155 Punkten.

Die Sekundarstufe II entspricht der Ausbildung zur Gymnasiallehrperson und wurde in der Schweiz nicht getestet (vgl. Kapitel 2 in diesem Band). Es zeigen sich hier mit einer Spannbreite von 240 Punkten sehr grosse Länderunterschiede. Die höchsten Leistungen in der Mathematikdidaktik werden wiederum in Taiwan erzielt (649 Punkte), gefolgt von Deutschland, Russland und Singapur mit einem deutlichen Abstand von mindestens 64 Punkten. Während das Ergebnis von Deutschland im Ausbildungstyp Sekundarstufe I signifikant niedriger ausfiel als in der Deutschschweiz, verfügen die angehenden Gymnasiallehrkräfte in Deutschland über ein signifikant höheres mathematikdidakti- 
sches Wissen als die angehenden Lehrpersonen der Sekundarstufe I in der Deutschschweiz.

Abbildung 11.14: Mathematikdidaktisches Wissen von angehenden Lehrpersonen der Sekundarstufe I im Ländervergleich

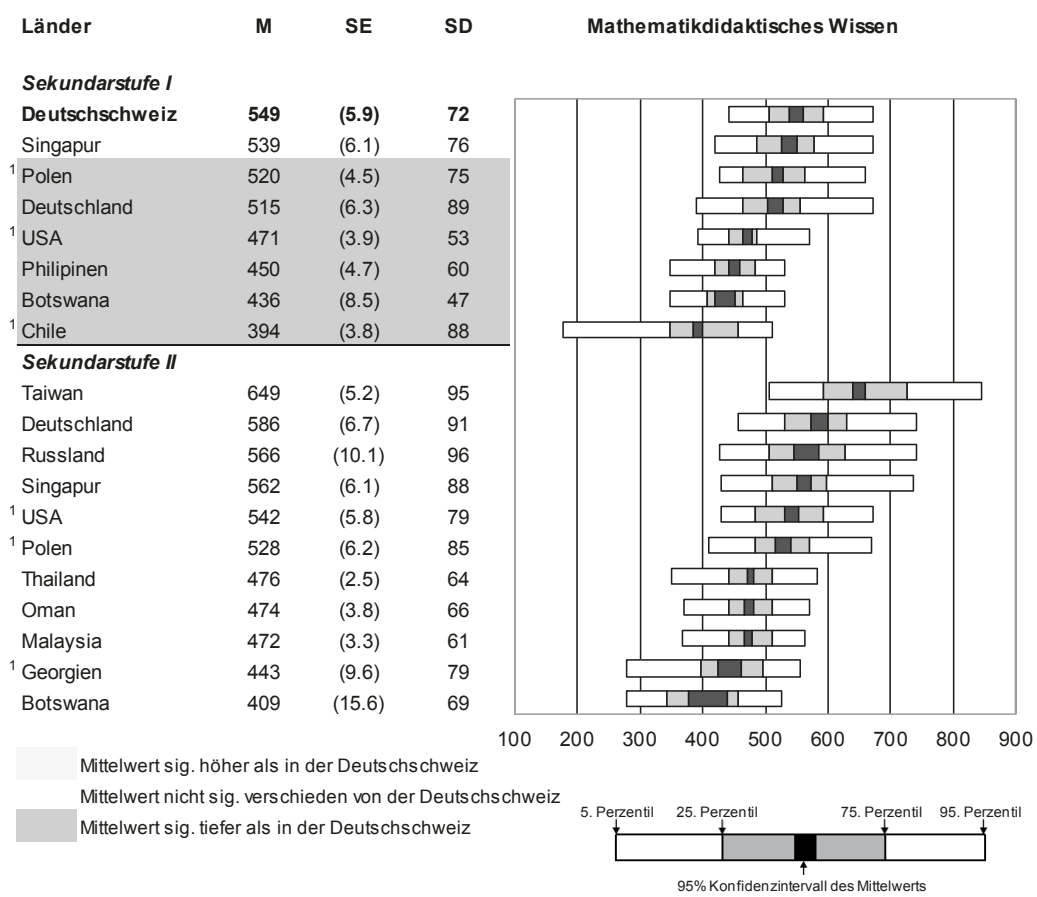

Anmerkungen: $\mathrm{M}=$ Mittelwert; $\mathrm{SE}=$ Standardfehler des Mittelwerts; $\mathrm{SD}=$ Standardabweichung. 1) USA, Polen, Georgien und Chile weisen eine Rücklaufquote von weniger als $75 \%$ auf.

Ergebnisse nach Leistungsniveaus in der Mathematikdidaktik

Wie bei der Primarstufe lassen sich die Leistungen der angehenden Lehrpersonen der Sekundarstufe I im mathematikdidaktischen Test einem von zwei Leistungsniveaus zuordnen, einem niedrigeren für Leistungen bis zu 509 Punkten und einem höheren für Leistungen über diesem Referenzwert (zur inhaltlichen Beschreibung von Leistungen um den Referenzwert vgl. Kapitel 11.2.2).

Abbildung 11.15 zeigt die Anteile der angehenden Lehrpersonen im jeweiligen Leistungsniveau für alle Teilnehmerländer. In der Deutschschweiz erreichen $71 \%$ der angehenden Lehrpersonen der Sekundarstufe I das höhere Leistungsniveau. Die Deutschschweiz hat auf der Sekundarstufe I, gefolgt von Deutschland, den höchsten Anteil angehender Lehrpersonen, die bei Studien- 
ende über ein hohes Leistungsniveau in Mathematikdidaktik verfügen. In Botswana und Chile erreichen weniger als 10\% der angehenden Lehrpersonen das höhere Leistungsniveau in Mathematikdidaktik.

Für die Sekundarstufe II liegt Taiwan mit 93\% der angehenden Lehrpersonen, welche über das höhere Leistungsniveau verfügen, an der Spitze. In Deutschland erreichen vier von fünf angehenden Gymnasiallehrpersonen das obere Leistungsniveau in Mathematikdidaktik. Botswana nimmt mit einem Anteil von 5\% im höheren Leistungsniveau den letzten Platz ein.

\section{Abbildung 11.15: Mathematikdidaktisches Wissen von angehenden Lehrpersonen der Sekundarstufe I nach Leistungsniveau}

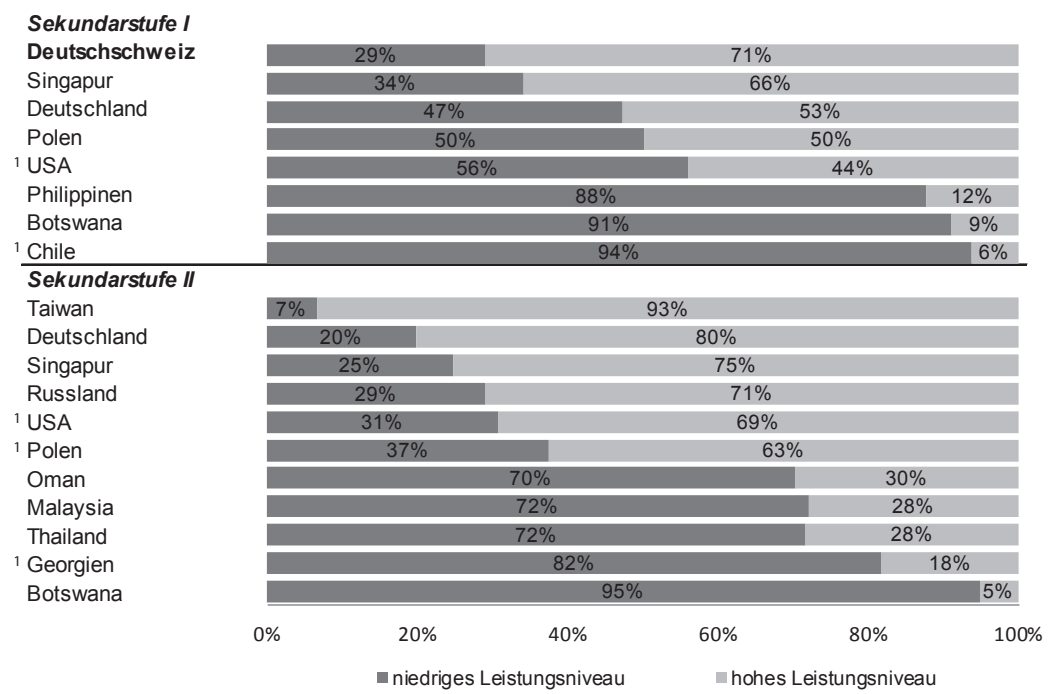

Anmerkungen: Sortierung nach Anteilen mit hohem Leistungsniveau.

1) USA, Polen, Georgien und Chile weisen eine Rücklaufquote von weniger als $75 \%$ auf.

\subsubsection{Mathematikdidaktisches Wissen nach Ausbildungsgängen in der Deutschschweiz}

Zwischen den Deutschschweizer Institutionen bzw. Ausbildungsgängen für angehende Lehrpersonen der Sekundarstufe I zeigt sich mit einer Differenz von 46 Punkten ein relativ homogenes Bild bezüglich des mathematikdidaktischen Wissens. Es sind keine statistischen Unterschiede zum Deutschschweizer Mittelwert oder zwischen den einzelnen Ausbildungsgängen zu finden. Es zeigt sich aber, dass die Streuungen innerhalb der Ausbildungsgänge teilweise sehr gross sind. Es gibt zwar zwei Ausbildungsgänge mit relativ homogenen 
Leistungen (S5, S1), die anderen Ausbildungsgänge weisen deutlich heterogenere Leistungen ihrer Abgängerinnen und Abgänger auf. Dies trifft insbesondere auf die Ausbildungsgänge S8 und S4 zu.

Abbildung 11.16: Mathematikdidaktisches Wissen von angehenden Lehrpersonen der Sekundarstufe I nach Ausbildungsgängen in der Deutschschweiz

$\begin{array}{lccc}\text { Ausbildungsgänge } & \mathbf{M} & \text { SE } & \text { SD } \\ \text { S8 } & 571 & (31.0) & 96 \\ \text { S1 } & 571 & (7.8) & 44 \\ \text { S4 } & 560 & (15.8) & 89 \\ \text { Deutschschweiz } & \mathbf{5 4 9} & \mathbf{( 5 . 9 )} & \mathbf{8 9} \\ \text { S6 } & 548 & (16.1) & 67 \\ \text { S3 } & 547 & (14.4) & 61 \\ \text { S5 } & 544 & (8.9) & 28 \\ \text { S2 } & 528 & (20.7) & 78 \\ \text { S7 } & 523 & (12.2) & 56\end{array}$

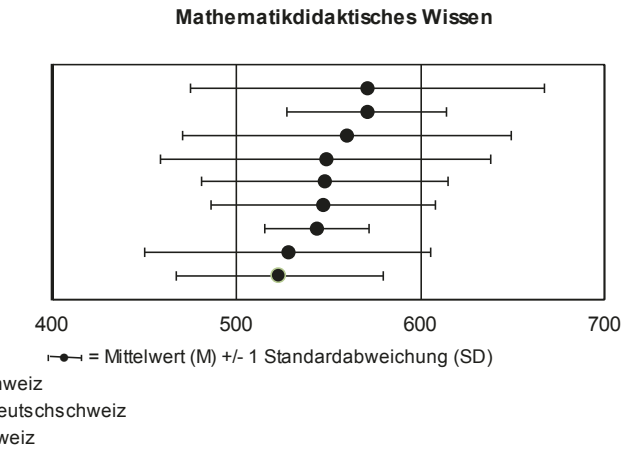

\subsubsection{Individuelle Merkmale und mathematikdidaktisches Wissen}

Geschlechterunterschiede im mathematikdidaktischen Wissen

Insgesamt sind die Geschlechterdifferenzen in der Mathematikdidaktik geringer als beim Fachwissen. Innerhalb des Ausbildungstyps Sekundarstufe I unterscheiden sich in der Mathematikdidaktik die Leistungen der Männer und Frauen in keinem Land signifikant (Abbildung 11.17). Dies gilt auch für die Deutschschweiz, wo der Leistungsvorsprung der Männer 18 Punkte beträgt.

Die einzigen signifikanten Geschlechterunterschiede finden sich bei den angehenden Lehrpersonen für die Sekundarstufe II. In Polen und Singapur schneiden die angehenden Lehrer in der Mathematikdidaktik um 31 bzw. 23 Punkte besser ab als die künftigen Lehrerinnen. In den übrigen Ländern zeigen sich keine geschlechtsspezifischen Unterschiede. 
Abbildung 11.17: Geschlechterunterschiede im mathematikdidaktischen Wissen von angehenden Lehrpersonen der Sekundarstufe im internationalen Vergleich

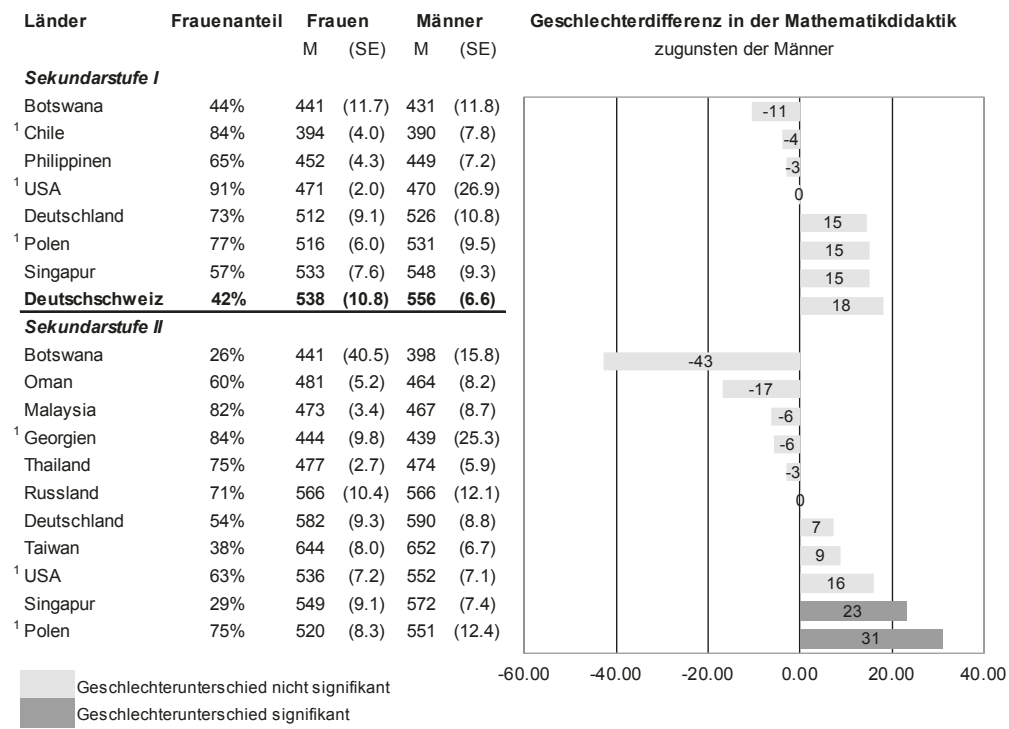

Anmerkungen: Die Länder sind nach Grösse des Geschlechterunterschieds innerhalb der beiden Ausbildungstypen sortiert.

1) USA, Polen, Chile und Georgien weisen eine Rücklaufquote von weniger als $75 \%$ auf.

Bedeutung individueller Merkmale für das mathematikdidaktische Wissen

Die gemeinsame Berücksichtigung verschiedener individueller Merkmale in einem multiplen Regressionsmodell bestätigt das oben dargestellte Ergebnis, dass in der Deutschschweiz bei angehenden Lehrpersonen der Sekundarstufe I kein signifikanter Geschlechterunterschied im mathematikdidaktischen Wissen besteht (Abbildung 11.18). Anders als bei der Primarstufe besteht bei der Sekundarstufe I auch kein Zusammenhang zwischen der selbstberichteten Leistungseinschätzung in der Sekundarschulzeit und der Leistung in Mathematikdidaktik.

Die soziale Herkunft hingegen hängt in der Deutschschweiz im Ausbildungstyp Sekundarstufe I negativ mit der mathematikdidaktischen Leistung zusammen. Dies bedeutet, Studierende aus weniger privilegierten sozialen Verhältnissen erreichen höhere Leistungen in der Mathematikdidaktik. Dieser leicht negative Zusammenhang könnte als Hinweis darauf interpretiert werden, dass begabte junge Menschen aus privilegierten Verhältnissen keine Leh- 
rerausbildung absolvieren, sondern eher ein anderes Studien- bzw. Berufsziel verfolgen (vgl. Kapitel 4 in diesem Band).

Abbildung 11.18: Vorhersage mathematikdidaktischen Wissens von angehenden Lehrpersonen der Sekundarstufe I durch individuelle Merkmale

Sekundarstufe I

Geschlecht (männlich)

Sozioökonomischer Hintergrund

Leistungseinschätzung

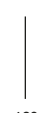

480
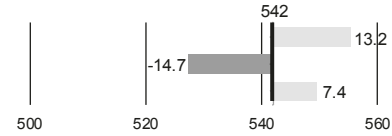

Mathematikdidaktisches Wissen

Anmerkung: Die Analysen erfolgten mittels multipler linearer Regression. Das Geschlecht ist dummycodiert (Referenzgruppe "weiblich"); die übrigen Prädiktoren sind z-standardisiert. Die Balkenlängen entsprechen den unstandardisierten Regressionskoeffizienten (B).

\subsubsection{Motivationale Orientierung und mathematikdidaktisches Wissen}

Die Zusammenhänge zwischen der Berufsmotivation und dem mathematikdidaktischen Wissen fallen auf der Sekundarstufe I insgesamt schwach aus. Einzig zwischen der fachbezogenen Motivation und der Leistung in der Mathematikdidaktik besteht ein signifikanter Zusammenhang. Angehende Sekundarlehrpersonen, die einen um eine Standardabweichung höheren Wert in der fachbezogenen Motivation aufweisen, erzielen 14 Punkte mehr im Mathematikdidaktiktest. Eine höhere Motivation, den Lehrberuf (auch) aus Interesse an der Mathematik auszuüben, scheint eine günstige Voraussetzung zu sein, um ein hohes mathematikdidaktisches Wissen zu erwerben.

Abbildung 11.19: Zusammenhänge zwischen motivationalen Orientierungen und mathematikdidaktischem Wissen bei angehenden Primarlehrpersonen in der Deutschschweiz

Sekundarstufe I

Interesse an Vermittlung

Interesse an Lernenden

Extrinsische Berufsmotivation

Fachbezogene Motivation

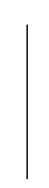

530

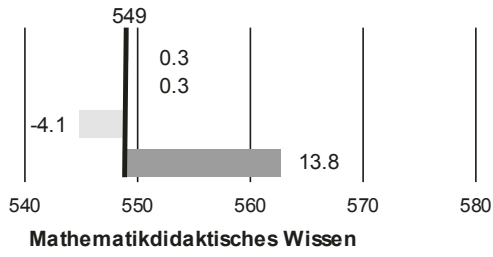

Zusammenhang nicht signifikant

Zusammenhang signifikant

Anmerkung: Die Analysen erfolgten mittels multipler linearer Regression. Die Balkenlängen entsprechen den unstandardisierten Regressionskoeffizienten (B). Alle Prädiktoren sind z-standardisiert. 


\section{$11.5 \quad$ Fazit}

Die Ergebnisse von TEDS-M zeichnen ein insgesamt positives Bild über die Leistungen der angehenden Lehrpersonen der Deutschschweiz in der Mathematikdidaktik. Am Ende ihrer Ausbildung verfügen im internationalen Vergleich sowohl die künftigen Lehrpersonen der Primarstufe als auch der Sekundarstufe I über ein vergleichsweise hohes mathematikdidaktisches Wissen.

Im internationalen Vergleich nimmt die Deutschschweiz auf der Unteren Primarstufe (1-4) mit einem Mittelwert von 519 Punkten den Spitzenplatz ein. Für die Ausbildung mit der Unterrichtsberechtigung bis zur sechsten Primarklasse steht die Deutschschweiz (539 Punkte) zusammen mit den USA im Mittelfeld. Die beiden ostasiatischen Länder Taiwan und Singapur erbringen jedoch erheblich bessere mathematikdidaktische Leistungen, Spanien und die Philippinen signifikant schlechtere. Auf der Sekundarstufe I nimmt die Deutschschweiz (549) zusammen mit Singapur den Spitzenplatz ein. Dieses Ergebnis wird jedoch von Taiwan als Spitzenreiter im Ausbildungstyp Sekundarstufe II noch um 100 Punkte übertroffen. Es zeigt sich, dass in der Deutschschweiz in allen Ausbildungstypen mehr als 75\% der angehenden Lehrpersonen über dem internationalen Durchschnitt liegen.

Aus einer deutschschweizerischen Perspektive betrachtet, sind folgende Befunde zum mathematikdidaktischen Wissen besonders zu erwähnen:

1. Die Leistungsunterschiede in der Mathematikdidaktik sind in der Deutschschweiz für beide Zielstufen deutlich geringer als in den relevanten Vergleichsländern. Dies bedeutet einerseits, dass zwar weniger Spitzenleistungen ausgewiesen werden, aber andererseits auch, dass der grosse Teil der angehenden Lehrpersonen Mindestanforderungen an fachdidaktischem Wissen in Mathematik erfüllt.

Im Vergleich der beiden deutschsprachigen Länder fällt auf, dass in Deutschland nicht nur das mathematische, sondern auch das mathematikdidaktische Wissen stark vom jeweiligen Ausbildungstyp abhängt. So erreichen in Deutschland die angehenden Primarlehrpersonen, die als Fachspezialistinnen und -spezialisten ausgebildet werden, vergleichbare Ergebnisse wie jene in der Deutschschweiz. Im nicht spezialisierten Ausbildungstyp (Primarstufe, 1-4) sind die mathematikdidaktischen Kenntnisse dagegen signifikant niedriger auch im Vergleich mit der Deutschschweiz.

Dies hat zur Folge, dass das fachdidaktische Wissen stark variiert und ein beträchtlicher Teil der Primarschulkinder von Lehrpersonen unterrichtet werden wird, die nur über ein rudimentäres Wissen in Mathematikdidaktik verfügen (Blömeke, Kaiser \& Lehmann, 2010b; 2010a). Aufgrund der hohen Bedeutung der Fachdidaktik für einen gelingenden Unterricht ist in Deutschland wie in der Schweiz anzustreben, den Anteil an Lehrpersonen mit sehr schwachen mathematikdidaktischen Leistungen weiter zu reduzie- 
ren bzw. alle künftigen Lehrpersonen auf ein bestimmtes Mindestniveau zu bringen.

2. Angehende Deutschschweizer Primarlehrpersonen, die eine Lehrberechtigung bis zur sechsten Jahrgangsklasse erwerben, verfügen nicht nur über ein höheres Mathematikwissen als angehende Lehrpersonen für die Unterstufe, sondern auch über ein höheres mathematikdidaktisches Wissen. Inwiefern dieser Befund durch bereits vorhandene Leistungsdifferenzen bei Studienbeginn, etwa durch bestimmte Selektionsmechanismen, erklärbar ist, oder ob die Differenzen zwischen den Ausbildungstypen erst im Verlauf der Ausbildung entstehen, ist Gegenstand weiterer Untersuchungen im Rahmen der schweizerischen Zusatzstudien zu TEDS-M.

3. Die angehenden Lehrpersonen der Sekundarstufe I in der Deutschschweiz erzielen in der Mathematikdidaktik eine um 18 Punkte höhere Leistung als in der Mathematik. Diese Differenz lässt sich zwar nicht unmittelbar vergleichen, weil die beiden Bereiche nicht auf einer gemeinsamen Skala abgebildet werden können. Im internationalen Vergleich lässt sich dieser Unterschied aber als relative Stärke der Deutschschweiz in der Mathematikdidaktik interpretieren. Auf der Primarstufe sind die Leistungen in der Mathematik und der Mathematikdidaktik, relativ zu den anderen Ländern, ausgeglichen.

4. Die Leistungsunterschiede zwischen den Deutschschweizer Lehrerbildungsinstitutionen in der Mathematikdidaktik fallen zwar bei den Ausbildungsgängen für die Primarstufe etwas geringer aus als beim mathematischen Wissen. Die maximale Mittelwertdifferenz von 82 Punkten ist dennoch beachtlich. Die absoluten Mittelwerte sind aber angesichts der teilweise kleinen Fallzahlen innerhalb der Ausbildungsgänge auch mit einer gewissen Unsicherheit behaftet. Möglicherweise hängen die etwas homogeneren Leistungen in der Mathematikdidaktik damit zusammen, dass Mathematikdidaktik anders als Mathematik an allen Institutionen Ausbildungsbestandteil ist und nicht davon ausgegangen wird, dass mathematikdidaktisches Wissen vor der Lehrerausbildung erworben worden ist.

Bei der Sekundarstufe I sind die Unterschiede zwischen den Institutionen mit maximal 46 Punkten wie schon beim Mathematikwissen deutlich geringer als bei der Primarstufe.

5. Am Ende der Lehrerausbildung in der Deutschschweiz bestehen keine signifikanten Geschlechterunterschiede im mathematikdidaktischen Wissen, weder für angehende Primarlehrpersonen noch für künftige Lehrpersonen der Sekundarstufe I. Offenbar wirkt sich der geringe, aber statistisch signifikante Rückstand der angehenden Primarlehrerinnen im mathematischen Wissen (vgl. Kapitel 10 in diesem Band) nicht auf die Mathematikdidaktik aus. Auf 
der Sekundarstufe I waren auch beim mathematischen Wissen keine Geschlechterdifferenzen festzustellen.

Bei der Primarstufenausbildung besteht auch zwischen der sozialen Herkunft und der Leistung in Mathematikdidaktik kein signifikanter Zusammenhang. Auf der Sekundarstufe I zeigt sich sogar ein leicht negativer ZusammenhangzwischendersozialenHerkunftunddenmathematikdidaktischen Leistungen. Diese Befunde könnten darauf hinweisen, dass die Lehrerausbildung bei Familien mit einem niedrigeren sozioökonomischen Hintergrund als Möglichkeit zum sozialen Aufstieg gilt (vgl. Kapitel 4 in diesem Band). Umgekehrt tendieren möglicherweise junge Menschen mit hohen Kompetenzen in einem familiären Umfeld mit einem hohen sozioökonomischen Status eher zu einer universitären Ausbildung.

6. Angehende Lehrpersonen mit einer höheren fachbezogenen Motivation, Lehrperson zu werden, erreichen höhere Leistungen in der Mathematikdidaktik. Dieser Zusammenhang lässt sich für beide untersuchten Zielstufen nachweisen.

Im Bereich der Mathematikdidaktik kann im internationalen Vergleich der Deutschschweizer Lehrerbildung ein gutes Zeugnis ausgestellt werden. Dies ist gerade auch deshalb bemerkenswert, weil aufgrund des geringen Spezialisierungsgrads der Lehrerausbildung in der Schweiz vergleichsweise wenig zeitliche Ressourcen für die Vermittlung von fachdidaktischen Inhalten in einzelnen Fächern zur Verfügung stehen. In zahlreichen anderen Ländern erwerben die angehenden Lehrpersonen auch auf der Primarstufe nur eine Lehrberechtigung für weniger Fächer und durchlaufen eine spezialisierte Ausbildung mit Schwerpunkt Mathematik, wie dies beispielsweise in Taiwan und Singapur, aber auch in Deutschland der Fall ist (vgl. Kapitel 10.5 in diesem Band).

Erklärungen für hohe Leistungen im Bereich der Mathematikdidaktik der angehenden Primar- und Sekundarlehrpersonen in der Deutschschweiz dürften neben den individuellen Voraussetzungen wie hohes mathematisches Wissen oder günstige motivationale Orientierungen auch auf der Ebene der Lehrerausbildungsinstitution selbst zu suchen sein. So finden sich bedeutsame Zusammenhänge zwischen dem Umfang der angebotenen Lerngelegenheiten in Mathematik und Mathematikdidaktik und dem mathematikdidaktischen Wissen (vgl. Kapitel 7 in diesem Band). Auch wenn sich dieses Ergebnis aufgrund der querschnittlichen Forschungsanlage nicht kausal interpretieren lässt, so erhärtet es doch die Annahme, dass gehaltvolle Lerngelegenheiten in der Lehrerbildung das Leistungsergebnis günstig beeinflussen.

Wie einleitend in diesem Kapitel ausgeführt, hat die Bedeutung der Fachdidaktik in den letzten Jahren zugenommen, nicht zuletzt deshalb, weil zunehmend empirisch belastbare Erkenntnisse vorliegen, die zeigen, dass dem fachdidaktischen Wissen eine zentrale Rolle für eine lernförderlichen Unterricht zukommt (Baumert et al., 2010; Baumert \& Kunter, 2011). Aus der hohen Bedeutung der Fachdidaktik folgt aber auch das Desiderat, mehr empirisch 
gesichertes Wissen über die Genese fachdidaktischer Kompetenzen in der Lehrerausbildung zu gewinnen, um dadurch die Qualität der fachdidaktischen Ausbildung weiterentwickeln zu können.

\subsection{Literatur}

Baumert, J. \& Kunter, M. (2011). Das mathematikspezifische Wissen von Lehrkräften, kognitive Aktivierung im Unterricht und Lernfortschritte von Schülerinnen und Schülern. In M. Kunter, J. Baumert, W. Blum, U. Klusmann, S. Krauss \& M. Neubrand (Hrsg.), Professionelle Kompetenz von Lehrkräften. Ergebnisse des Forschungsprogramms COACTIV, (S. 163-192). Münster: Waxmann.

Baumert, J., Kunter, M., Blum, W., Brunner, M., Voss, T., Jordan, A., Klusmann, U., Krauss, S., Neubrand, M. \& Tsai, Y.-M. (2010). Teachers' mathematical knowledge, cognitive activation in the classroom, and student progress. American Educational Research Journal, 47(1), 133-180.

Blömeke, S., Kaiser, G. \& Lehmann, R. (Hrsg.) (2010a). TEDS-M 2008: Professionelle Kompetenz und Lerngelegenheiten angehender Mathematiklehrkräfte für die Sekundarstufe I im internationalen Vergleich. Münster: Waxmann.

Blömeke, S., Kaiser, G. \& Lehmann, R. (Hrsg.) (2010b). TEDS-M 2008: Professionelle Kompetenz und Lerngelegenheiten angehender Primarstufenlehrkräfte im internationalen Vergleich. Münster: Waxmann.

Blömeke, S., Suhl, U., Kaiser, G. \& Döhrmann, M. (2012). Family background, entry selectivity and opportunities to learn: What matters in primary teacher education? An international comparison of fifteen countries. Teaching and Teacher Education, 28(1), 44-55.

Brühwiler, C. (2014). Adaptive Lehrkompetenz und schulisches Lernen. Effekte handlungssteuernder Kognitionen von Lehrpersonen auf Unterrichtsprozesse und Lernergebnisse der Schülerinnen und Schüler. Münster: Waxmann.

Darling-Hammond, L. \& Youngs, P. (2002). Defining „Highly Qualified Teachers": What Does „Scientifically-Based Research“" Actually Tell Us? Educational Researcher, 31(9), 13-25.

Döhrmann, M., Kaiser, G. \& Blömeke, S. (2010). Messung des mathematischen und mathematikdidaktischen Wissens. In S. Blömeke, G. Kaiser \& R. Lehmann (Hrsg.), TEDS-M 2008. Professionelle Komopetenz und Lerngelegenheiten angehender Primarstufenlehrkräfte im internationalen Vergleich, (S. 169-179). Münster: Waxmann.

Fan, L. \& Cheong, N. P. C. (2002). Investigating the sources of Singaporean mathematics teachers' pedagogical knowledge. In D. Edge \& B. H. Yap 
(Hrsg.), Mathematics education for a knowledge-based era 2, (S. 224-231). Singapore: AME.

Heitzmann, A. (2013). Entwicklung und Etablierung der Fachdidaktik in der schweizerischen Lehrerinnen- und Lehrerbildung: Überlegungen zu Rolle und Bedeutung, Analyse des Ist-Zustands und Reflexionen für eine produktive Weiterentwicklung Beiträge zur Lehrerbildung, 31(1), 6-17.

Heitzmann, A., Tremp, P., Brühwiler, C., Niggli, A., Pauli, C., Reusser, K. \& Tettenborn, A. (2013). Editorial 1/2013. Beiträge zur Lehrerbildung, $31(1), 3-5$.

Helmke, A. (2003). Unterrichtsqualität. Erfassen, bewerten, verbessern. Seelze: Kallmeyer.

Kunter, M., Baumert, J., Blum, W., Klusmann, U., Krauss, S. \& Neubrand, M., (Hrsg.). (2011). Professionelle Kompetenz von Lehrkräften. Ergebnisse des Forschungsprogramms COACTIV. Münster: Waxmann.

Kunter, M. \& Klusmann, U. (2010). Die Suche nach dem kompetenten Lehrer - ein personenzentrierter Ansatz. In W. Bos, E. Klieme \& O. Köller (Hrsg.), Schulische Lerngelegenheiten und Kompetenzentwicklung, (S. 207-230). Münster: Waxmann.

Parchmann, I. (2013). Wissenschaft Fachdidaktik - eine besondere Herausforderung. Beiträge zur Lehrerbildung, 31(1), 31-41.

Shulman, L. S. (1987). Knowledge and teaching: Foundations of the new reform. Harvard Educational Review, 57(1), 1-22.

Tatto, M. T., Schwille, J., Senk, S. L., Ingvarson, L., Peck, R. \& Rowley, G. (2008). Teacher Education and Development Study in Mathematics (TEDS-M). Policy, practice, and readiness to teach primary and secondary mathematics. Conceptual framework. East Lansing, MI: Teacher Education and Development International Study Center, College of Education, Michigan State University.

Tatto, M. T., Schwille, J., Senk, S. L., Ingvarson, L., Rowley, G., Peck, R., Bankov, K., Rodriguez, M. \& Reckase, M. (2012). Policy, Practice, and Readiness to Teach Primary and Secondary Mathematics in 17 Countries. Findings from the IEA Teacher Education and Development Study in Mathematics (TEDS-M). Amsterdam: IEA.

Vollrath, H.-J. (2001). Grundlagen des Mathematikunterrichts in der Sekundarstufe. Heidelberg: Spektrum. 
